\title{
An In Vitro Study of Effect of Water Soluble Vitamin B Complex on Antimicrobial Activity of Antibiotics against Klebsiella species Isolated from Respiratory Samples
}

\author{
Sowmya Nasimuddin*, Jeevan Malaiyan, Mohanakrishanan Kandaswamy, \\ Swathithra Mohandass Nirmaladevi and Sumathi Gnanadesikan
}

\author{
Department of Microbiology, Sri Muthukumaran Medical College Hospital and Research \\ Institute, Chikkarayapuram, Chennai - 600069, Tamil Nadu, India \\ *Corresponding author
}

K e y w o r d s
Antimicrobial
activity of Vitamin
B complex,
Klebsiella
pneumoniae.

\section{Introduction}

Vitamins are essential for various biological responses in the human body ${ }^{(1)}$. Vitamins play an important role in conferring immunity, which protects against infection $(2,3)$. Vitamin B complex includes Thiamine $\left(\mathrm{B}_{1}\right)$, Riboflavin $\left(\mathrm{B}_{2}\right)$, Niacin $\left(\mathrm{B}_{3}\right)$, Pyridoxine $\left(\mathrm{B}_{6}\right)$, Pantothenic acid $\left(\mathrm{B}_{5}\right)$, Biotin $\left(\mathrm{B}_{7}\right)$ and Cyanocobalamine $\left(\mathrm{B}_{12}\right)$.Various studies analysed the interactions of antimicrobial agents with other drugs, food products, vitamin $\mathrm{K}$, vitamin $\mathrm{C}$ and vitamin $\mathrm{E}^{(3,4)}$.
No previous invitro studies are there to identify the effectiveness of water soluble vitamins as supplements (particularly vitamin B complex) with antimicrobials agents. Being the most commonly prescribed supplements in respiratory tract infections, this study was undertaken to find out the effect of water soluble vitamin B complex against antibacterial prescribed for respiratory infections. The infections of respiratory tract are quite common in adults and children 
worldwide, with Klebsiella pneumoniae being one of the important pathogen ${ }^{(5,6) .}$ Hence in this study, we proposed to find out the in vitro effect of water soluble vitamin B complex on antibacterial activity of various antibiotics using phenotypic disc diffusion methods.

\section{Materials and Methods}

Institutional ethical committee approval was obtained prior to the study. A prospective experimental study was carried out with 50 isolates of Klebsiella pneumoniae isolated from respiratory samples. The sputum samples requested for bacterial culture were processed in the laboratory. The Klebsiella pneumoniae were identified and isolated from respiratory samples and identified by standard laboratory procedures. The standardised antibiotics (HIMEDIA) used in this study were - Ciprofloxacin $(5 \mu \mathrm{g} / \mathrm{disc})$, Doxycycline $(30 \mu \mathrm{g} / \mathrm{disc}), \quad$ Gentamicin $\quad(10 \mu \mathrm{g} / \mathrm{disc})$, Imipenem $\quad(10 \mu \mathrm{g} / \mathrm{disc}), \quad$ Piperacillintazobactam $(100 / 10 \mu \mathrm{g} / \mathrm{disc}), \quad$ Ceftazidime $(30 \mu \mathrm{g} / \mathrm{disc})$, Ceftazidime - Clavulanic acid $(30 / 10 \mu \mathrm{g} / \mathrm{disc})$ and Chloramphenicol $(30 \mu \mathrm{g} / \mathrm{disc})$. Commercially available solutions of Vitamin B complex were obtained, with each $\mathrm{ml}$ containing Thiamine-mononitrate10mg, Pyridoxine-3mg, Riboflavin-10mg, Cyanocobalamin- $15 \mu \mathrm{g}$, Nicotinamide- $45 \mathrm{mg}$, Calcium Pantothenate-50mg. Two plates of sensitivity pattern was done for Klebsiella pneumoniae and recorded as per standard Clinical Laboratory Standards Institute (CLSI) guidelines ${ }^{(7)}$. To one plate $10 \mu \mathrm{l}$ of Vitamin B complex solution was pipetted out to each of the antibiotic discs and incubated at $35 \pm 2^{\circ} \mathrm{C}$, for 24 hours ${ }^{(7)}$. The readings of this plate were taken as indicative of combination of Vitamin B complex and Antibiotics.

The diameter of the inhibition zones were measured in millimetre after 24 hours of incubation using a standard scale. The zone diameter of individual antibiotics in sensitivity pattern testing was kept as control, and the zone diameter of antibiotics in combination with Vitamin B complex were recorded. Any increase, decrease or no change in zone size of vitamins and antibiotic combination in comparison with individual antibiotics (control) were noted. The differences in zones were statistically analysed by student $t$ test for significance.

\section{Results and Discussion}

The antibiotic sensitivity of 50 Klebsiella pneumoniae showed that, most of the isolates were sensitive to antibiotics, Chloramphenicol (84\%), Doxycycline (68\%), Gentamicin (68\%) and Ciprofloxacin (64\%). Most resistance was observed along the antibiotics, Ceftazidime $(48 \%)$ and PiperacillinTazobactam (44\%). In our study 6 isolates of Klebsiella pneumoniae were found to be extended spectrum betalactamase (ESBL). The complete sensitivity patterns of isolates were illustrated in Figure 1. It was observed that there were differences in zone diameter on addition of Vitamin B complex to the antibiotics. The number of isolates showing increases, decreases and no change are depicted for each antibiotic in figure 2. Major increase in zone was shown along with Imipenem (24 isolates) and Piperacillin tazobactam (24 isolates). Decrease of zone diameter was mainly noted along with Doxycycline (26 isolates) and Chloramphenicol (22 isolates) (Figure 3).

Herewith, we tend to explain the changes noted with Ciprofloxacin with and without vitamin B complex solution. On susceptibility testing for Ciprofloxacin, 32 isolates were sensitive, out of which on addition of Vitamin B complex solution, 8 isolates showed increase in zone, 14 isolates showed decrease of zone and 10 isolates did not show any change in zone. On addition of Vitamin B complex, out of the 14 resistant isolates, 4 
isolates showed decrease in zone and 10 isolates showed no change in zone. There were 4 isolates with intermediate zone size, of which 2 showed increase in zone size, whereas 2 remain the same. The increase in zone ranged from $2-8 \mathrm{~mm}$ and decrease in zone ranged from $1-5 \mathrm{~mm}$. Neither the increase nor the decrease was significant on analysis by student $t$ test. The details of the above observations along with other antibiotics have been illustrated in Table 1 for all the antibiotics.

On testing with Ceftazidime-Clavulanic acid and Ceftazidime, 6 ESBL isolates were noted.
On interactions with Vitamin B complex, the increase ranged from $1-8 \mathrm{~mm}$ and decrease ranged from $1-10 \mathrm{~mm}$. The decrease noted was statistically significant by student $t$ test.

As per student $\mathrm{t}$ test analysis, significant increase in zone diameter was noted addition of Vitamin B complex to antibiotics Imipenem (1-8mm by 24 isolates) and Piperacillin-tazobactam $\quad(1-9 \mathrm{~mm}$ by 24 isolates). Significant decrease in zone diameter was noted on addition of Vitamin B complex to antibiotics Chloramphenicol (1$7 \mathrm{~mm}$ by 22 isolates) and CeftazidimeClavulanic acid (1-10mm by 18 isolates).

Table.1 Vitamin B complex and antibiotics

\begin{tabular}{|c|c|c|c|c|c|c|c|}
\hline Antibiotic & Isolates/50 & \multicolumn{6}{|c|}{ VITAMIN B COMPLEX } \\
\hline \multirow{4}{*}{ Ciprofloxacin } & \multirow{3}{*}{$\begin{array}{l}\text { SENSITIVE-32 } \\
\text { INTERMED-4 }\end{array}$} & \multirow{4}{*}{\multicolumn{2}{|c|}{$\begin{array}{l}\text { INCREASE } \\
2-8 \mathrm{~mm} \\
\text { NOT } \\
\text { SIGNIFICANT } \\
\end{array}$}} & \multirow{4}{*}{\multicolumn{2}{|c|}{\begin{tabular}{|l|l}
\multicolumn{2}{|c}{ DECREASE } \\
14 & $1-5 \mathrm{~mm}$ \\
- & NOT \\
\cline { 1 - 1 } & SIGNIFICANT \\
\end{tabular}}} & \multicolumn{2}{|r|}{ SAME } \\
\hline & & & & & & 10 & NOT \\
\hline & & & & & & 2 & SIGNIFICANT \\
\hline & RESISTANT-14 & & & & & 10 & \\
\hline Doxycycline & SENSITIVE-34 & 10 & $2-6 \mathrm{~mm}$ & 20 & $1-7 \mathrm{~mm}$ & 4 & NOT \\
\hline & INTERMED-4 & 2 & NOT & 2 & NOT & - & SIGNIFICANT \\
\hline & RESISTANT-12 & - & SIGNIFICANT & 4 & SIGNIFICANT & 8 & \\
\hline Gentamicin & SENSITIVE-34 & 10 & $1-8 \mathrm{~mm}$ & 20 & $1-4 \mathrm{~mm}$ & 4 & NOT \\
\hline & INTERMED-2 & 2 & NOT & - & NOT & - & SIGNIFICANT \\
\hline & RESISTANT-14 & 6 & SIGNIFICANT & - & SIGNIFICANT & 8 & \\
\hline Imipenem & SENSITIVE-18 & 4 & $1-8 \mathrm{~mm}$ & 14 & $2-8 \mathrm{~mm}$ & - & NOT \\
\hline & INTERMED-20 & 12 & SIGNIFICANT & 4 & NOT & 4 & SIGNIFICANT \\
\hline & RESISTANT-12 & 8 & & 2 & SIGNIFICANT & 2 & \\
\hline PIT & SENSITIVE-8 & 2 & $1-9 \mathrm{~mm}$ & 4 & $1-5 \mathrm{~mm}$ & 2 & NOT \\
\hline & INTERMED-20 & 14 & SIGNIFICANT & 6 & NOT & - & SIGNIFICANT \\
\hline & RESISTANT-22 & 8 & & 6 & SIGNIFICANT & 8 & \\
\hline Ceftazidime & SENSITIVE-18 & 4 & $1-4 \mathrm{~mm}$ & 6 & $1-15 \mathrm{~mm}$ & 8 & NOT \\
\hline & INTERMED-8 & 6 & NOT & 2 & NOT & - & SIGNIFICANT \\
\hline & RESISTANT-24 & - & SIGNIFICANT & 8 & SIGNIFICANT & 16 & \\
\hline Ceftazidime & ESBL-6 & 4 & $1-8 \mathrm{~mm}$ & 2 & $1-10 \mathrm{~mm}$ & - & NOT \\
\hline $\begin{array}{l}\text { Clavulanic } \\
\text { acid }\end{array}$ & NON ESBL-44 & 14 & $\begin{array}{l}\text { NOT } \\
\text { SIGNIFICANT }\end{array}$ & 16 & SIGNIFICANT & 14 & SIGNIFICANT \\
\hline Chloramphe & SENSITIVE-42 & 10 & $1-4 \mathrm{~mm}$ & 20 & $1-7 \mathrm{~mm}$ & 12 & NOT \\
\hline -nicol & INTERMED-4 & - & NOT & - & SIGNIFICANT & 4 & SIGNIFICANT \\
\hline & RESISTANT-4 & 2 & SIGNIFICANT & 2 & & - & \\
\hline
\end{tabular}


Fig.1 Showing sensitivity pattern of Klebsiella pneumoniae

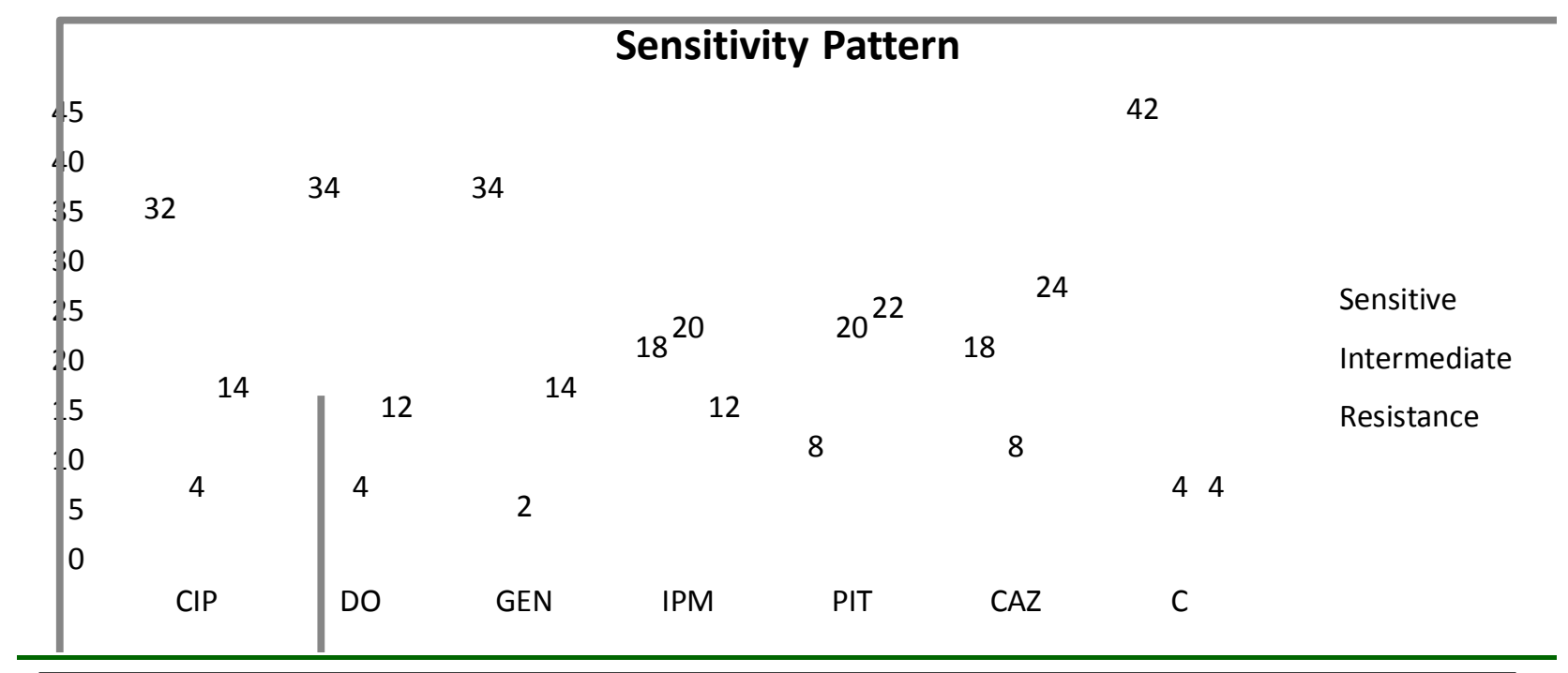

CIP-Ciprofloxacin; DO-Doxycycline; GEN-Gentamicin; IMP-Imipenem; PIT-Piperacillin tazobactam; CAZ-Ceftazidime; C-Chloramphenicol

Fig.2 Showing effect of Vitamin B complex on antibiotics

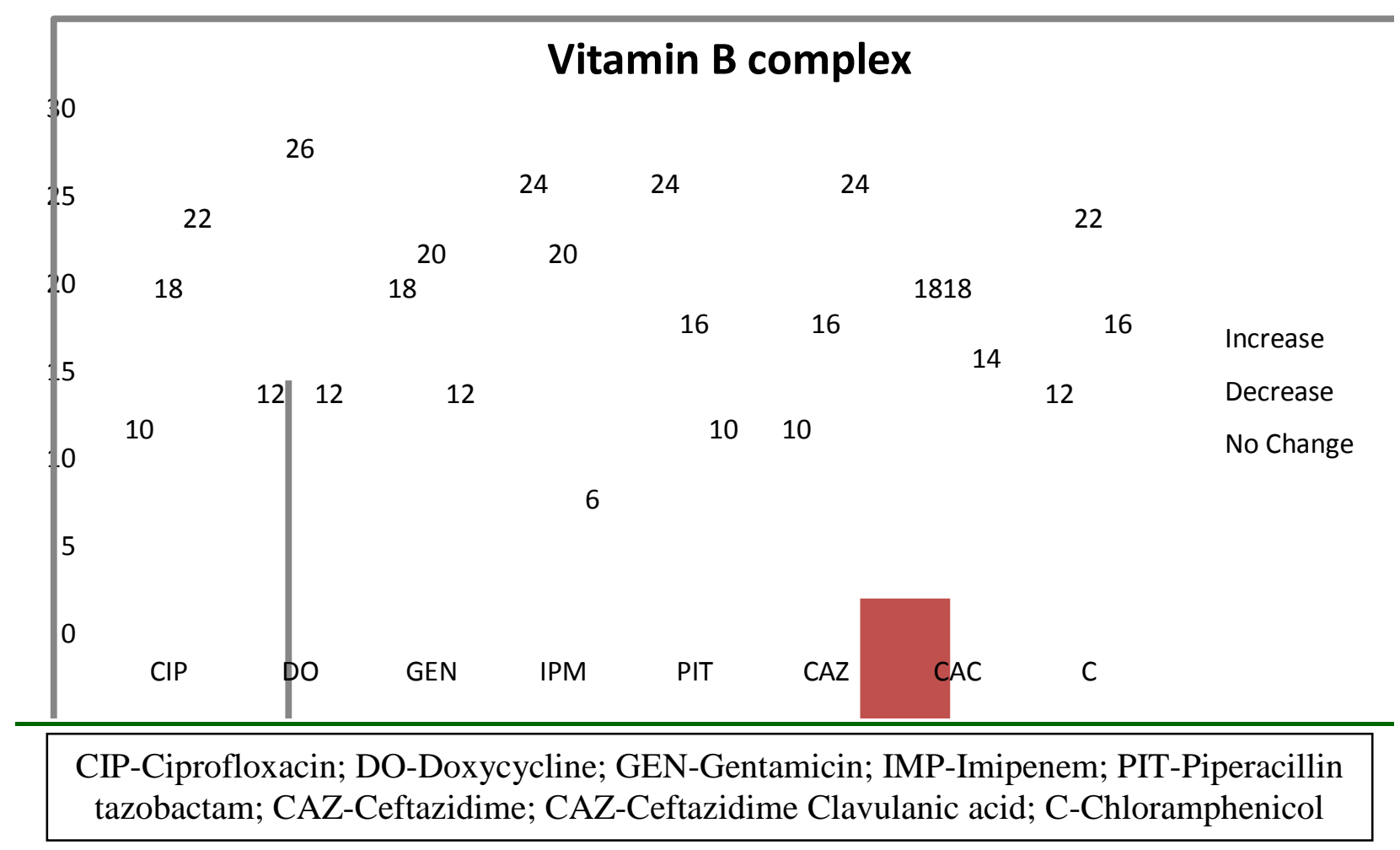

As depicted in figure 2, on addition of Vitamin B to ciprofloxacin, 10 isolates 
Fig.3 Sensitivity pattern with standard antibiotics and effect on addition of Vitamin B complex

a) Antibiotics alone

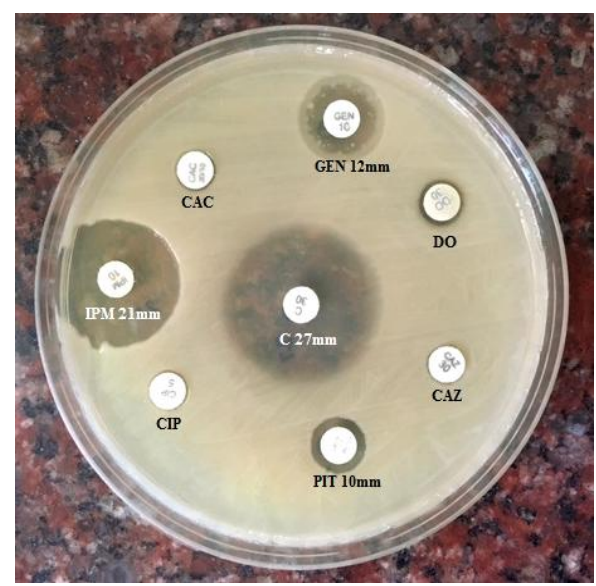

b) Vitamin B complex + antibiotics

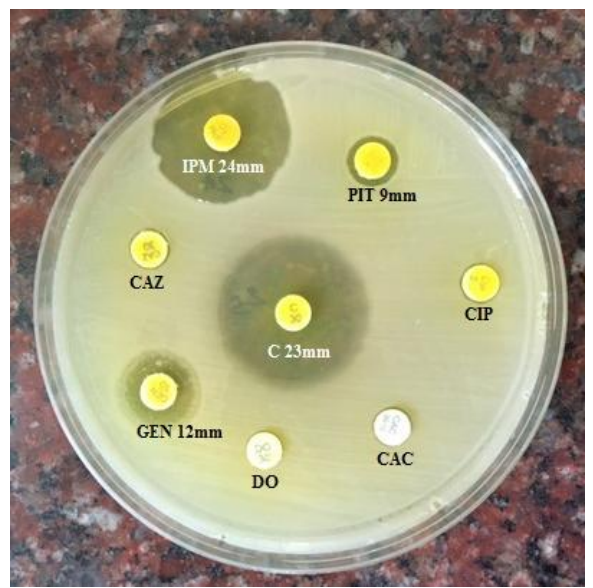

a) Sensitivity pattern observed with antibiotics against Klebsiella pneumoniae

b) Sensitivity pattern on addition of Vitamin B complex solution to antibiotics.

As noted in figure 3, on addition of Vitamin B complex, there is decrease in zone along with Chloramphenicol and Piperacillin tazobactam. While there increase in zone with Imipenem; no change is noted along with other antibiotics.

CIP-Ciprofloxacin; DO-Doxycycline; GEN-Gentamicin; IMP-Imipenem; PIT-Piperacillin tazobactam; CAZ-Ceftazidime; CAC-Ceftazidime Clavulanic acid; C-Chloramphenicol.

Klebsiella pneumoniae, one of the important pathogen causing respiratory infections, was isolated and studied for effects of water soluble vitamins on antibacterial activity of antibiotics. 50 isolates were taken; their sensitivity pattern was noted based on which treatment would be advised. It was noted that 6 isolates out of 50 were ESBL producing, indicating presence of drug resistant isolates.

On addition of water soluble vitamins to antibiotics and observing the differences in inhibition zone diameters by disc diffusion methods, there existed significant variations and interactions, in vitro. Vitamin B complex based on significant observations increases the inhibitory zone of Imipenem and Piperacillin- tazobactam; this indicates that Vitamin B complex enhances the antibacterial effect of Imipenem (48\%) and Piperacillin- tazobactam (48\%). In the same isolates, significant decrease of inhibitory zone was observed with Ceftazidime-Clavulanic acid (36\%) and Chloramphenicol (44\%); this indicates that Vitamin B complex decreases the antibacterial effect of Chloramphenicol and Ceftazidime-Clavulanic acid. The observations noted in this study need further development in terms of increased number of isolates and proper standardisation of Vitamin concentrations, with respect to each antibiotic. Studies can be done to compare the effect of Vitamins on antibiotics against other microorganisms also.

\section{Acknowledgements}

We would like to thank Indian Council of Medical Research (ICMR) for approving this project under Short Term Studentship - 2016. 


\section{References}

1.Chandra RK. Nutrition and the immune system from birth to old age. European Journal of Clinical Nutrition. 2002. 56(Suppl.3); S73-S76.

2.Vitamin and Mineral requirements in Human Nutrition: Report of a joint FAO/WHO expert consultation, Bangkok, Thailand, September 1998.

3.Fondell E, Bälter O, Rothman KJ. Dietary intake and Supplement use of Vitamins $\mathrm{C}$ and $\mathrm{E}$ and Upper respiratory tract infection. JAmColl Nutr 2011 Aug; 30(4):248-58.

4.Wang Y, Jia XM, Jia JH, Li MB, Cao YY, $\mathrm{Gao} \mathrm{PH}$ et al. Ascorbic acid decreases the antifungal effect of fluconazole in the treatment of candidiasis. Clin Exp Pharmacol Physiol. 2009 Oct; 36(10):e40-6.
5.A. S. Sikarwar and H. V. Batra. Identification of Klebsiella pneumoniae by Capsular Polysaccharide Polyclonal Antibodies. International Journal of Chemical Engineering and Applications, Vol. 2, No. 2, April 2011.

6.Asati Rakesh Kumar. Antimicrobial Sensitivity Pattern of Klebsiella pneumoniae isolated from Sputum from Tertiary Care Hospital, Surendranagar, Gujarat and Issues Related to the Rational Selection of Antimicrobials. Sch. J. App. Med. Sci., 2013; 1(6):928933.

7.Clinical Laboratory and Standards Institute. Performance standards for Antimicrobial Susceptibility Testing; Twenty second informational supplement. Approved standard M100-S15, 2012.

\section{How to cite this article:}

Sowmya Nasimuddin, Jeevan Malaiyan, Mohanakrishanan Kandaswamy, Swathithra Mohandass Nirmaladevi and Sumathi Gnanadesikan. 2017. An In Vitro Study of Effect of Water Soluble Vitamin B Complex on Antimicrobial Activity of Antibiotics against Klebsiella species Isolated from Respiratory Samples. Int.J.Curr.Microbiol.App.Sci. 6(10): 2782-2787. doi: https://doi.org/10.20546/ijcmas.2017.610.326 